\title{
Clinical and Imaging Characteristics of Coronavirus Disease 2019 Caused by SARS-CoV-2
}

\author{
Wendong Hao ${ }^{1}$ and Yunqing Zhang ${ }^{1}$ \\ ${ }^{1}$ Affiliation not available
}

May 5, 2020

\begin{abstract}
Since end of December 2019, a cluster of patients with pneumonia of unknown origin was reported from Wuhan, Hubei province, China. They shared a connection with the Huanan South China Seafood Market in Wuhan, and now it has been confirmed that the disease is caused by a novel coronavirus (officially named COVID-19). According to real-time statistics from Johns Hopkins University in the United States, as of 5:30 on the 31st, Beijing time, the number of confirmed cases of COVID-19 pneumonia in the world has exceeded 770,000, reaching 777,286, and the number of deaths has reached 37,140. Currently, clinicians have found some atypical cases with positive chest CT findings may present with negative results of RT-PCR for COVID-19. The timely diagnosis, isolation and treatment of these patients will help control the further spread of COVID-19.
\end{abstract}

\section{Hosted file}

Manuscript 2020-03-31.doc available at https://authorea.com/users/307338/articles/438279clinical-and-imaging-characteristics-of-coronavirus-disease-2019-caused-by-sars-cov-2

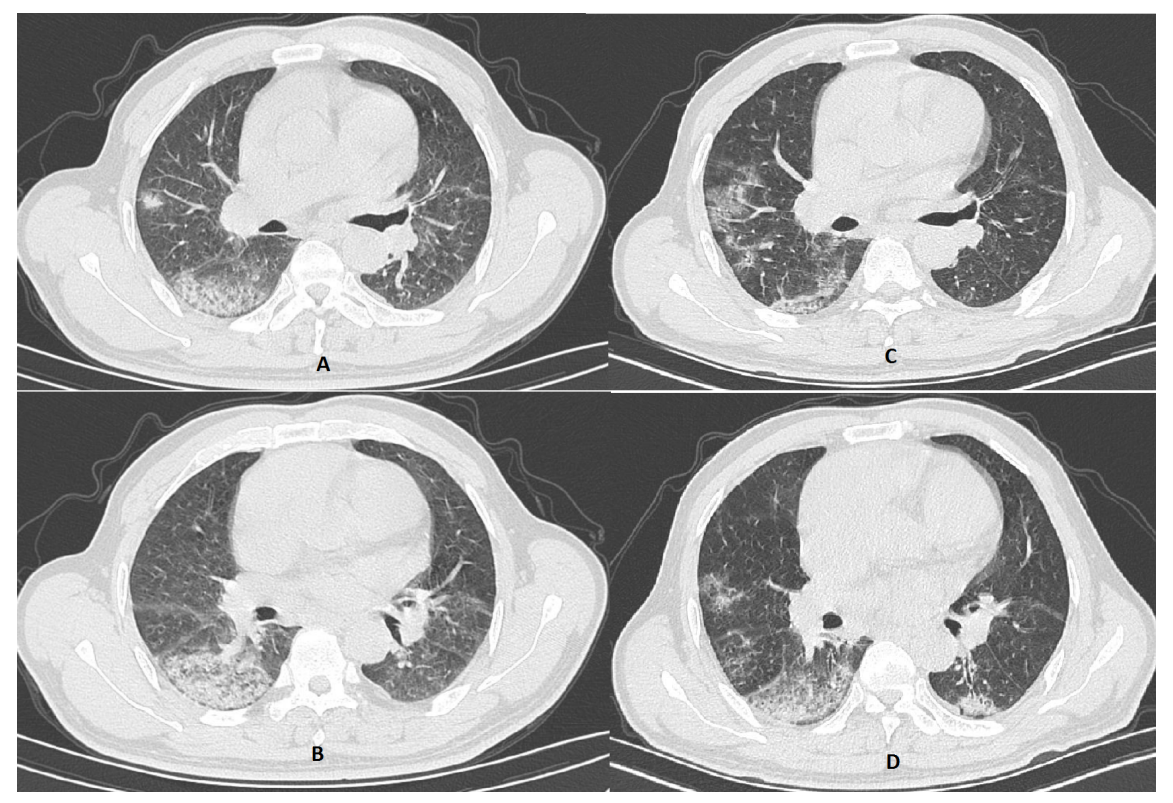

